Published in final edited form as:

Contraception. 2019 January ; 99(1): 22-26. doi:10.1016/j.contraception.2018.08.014.

\title{
Beyond intent: Exploring the association of contraceptive choice with questions about Pregnancy Attitudes, Timing and How important is pregnancy prevention (PATH) questions
}

\author{
Claudia Geist $^{1}$, Abigail RA Aiken ${ }^{2}$, Jessica N. Sanders ${ }^{1}$, Bethany G. Everett ${ }^{1}$, Kyl Myers $^{1}$, \\ Patty Cason ${ }^{3}$, Rebecca G. Simmons ${ }^{1}$, and David K. Turok ${ }^{1}$ \\ ${ }^{1}$ The University of Utah \\ ${ }^{2}$ University of Texas at Austin \\ ${ }^{3}$ UCLA School of Nursing
}

\section{Abstract}

Objectives-To explore women's responses to PATH questions (Pregnancy Attitudes, Timing and How important is pregnancy prevention) about hypothetical pregnancies and associations with contraceptive method selection among individuals who present as new contraceptive clients and desire to prevent pregnancy for at least one year.

Study Design-The HER Salt Lake Contraceptive Initiative provided no-cost contraception to new contraceptive clients for one year at family planning health centers in Salt Lake County. Those who wanted to avoid pregnancy for at least one-year and completed the enrollment survey are included in the current study. We used Poisson regression to explore the association between survey adapted PATH questions and contraceptive method selection.

Results-Based on an analytic sample of 3,121 individuals, we found pregnancy timing and happiness about hypothetical pregnancies to be associated with method selection. Clients who report plans to wait more than five years (Prevalence Rates (PR) 1.14; 95\% Confidence Interval (CI) 1.05-1.24), those who never wanted to become pregnant (PR 1.16; 95\% CI 1.07-1.26), or those who were uncertain $(\mathrm{PR}=1.19 ; 95 \%$ CI 1.09-1.30) were all more likely to select IUDs and implants than women who reported wanting to become pregnant within 5 years. Greater happiness was associated with lower chance of choosing an IUD or implant (PR 0.98; 95\% CI 0.96-0.999). Expressed importance of pregnancy prevention was not significantly associated with any specific contraceptive choice.

Corresponding Author: Claudia Geist; University of Utah Department of Sociology, 380 S. 1530 E. Room 301, Salt Lake City, UT, 84112; Claudia.geist@ soc.utah.edu, (801) 581-6170 (office); (801) 585-5146.

Publisher's Disclaimer: This is a PDF file of an unedited manuscript that has been accepted for publication. As a service to our customers we are providing this early version of the manuscript. The manuscript will undergo copyediting, typesetting, and review of the resulting proof before it is published in its final citable form. Please note that during the production process errors may be discovered which could affect the content, and all legal disclaimers that apply to the journal pertain.

Conflict of Interest:

The University of Utah Department of Obstetrics and Gynecology Program in Family Planning receives research funding from Bayer Women's Health Care, Merck \& Co. Inc., Teva Pharmaceuticals, Bioceptive, Contramed, and Medicines 360. PC originally developed the PATH questions and serves on an advisory Board for Teva, ContraMed, and Cooper Surgical and as speaker/trainer for Teva, ContraMed, and Merck. The other authors have no conflicts of interest to report. 
Conclusions-Pregnancy intentions and happiness about a hypothetical pregnancy were independently associated with selection of IUDs and Implants.

Implications-Pregnancy attitudes, plans and emotions inform clients' contraceptive needs and behaviors. Client-centered contraceptive care may benefit from a more nuanced PATH approach rather than relying on a single time-oriented question about pregnancy intention.

\section{Keywords}

pregnancy intentions; contraceptive method choice; emotions about pregnancy; cost barrier; LARC; PATH questions

\section{Introduction}

Pregnancy preferences are complex, nuanced, and subject to change over time [1-3]. A better understanding of the relationship between pregnancy preferences and contraceptive method choice is clinically relevant. Clinical tools, such as One Key Question ${ }^{\mathrm{TM}}$ have been developed to support the inclusion of pregnancy intention as a key health indicator in preventive care settings. One Key Question [4] asks clients "Would you like to become pregnant in the next year?" However, given the complexity of pregnancy preferences, the use of timing alone might not be sufficient to understand a patient's contraceptive needs and pregnancy intentions. PATH, which stands for Pregnancy Attitudes, Timing, How important is prevention [5], was originally a conversation guide to support client-centered contraceptive counseling and preconception care in the clinical setting. Key components of the PATH clinical tool include: prompts about patient pregnancy intentions; questions about whether and when pregnancy might be desired; and questions about the importance of preventing a pregnancy and happiness about a hypothetical pregnancy.

Apparent contradictions between timing-based pregnancy intentions and emotional orientations towards pregnancy (i.e. professing happiness about a pregnancy that would be unintended) have traditionally been viewed as ambivalence about avoiding conception [6,7]. Previous research has found that individuals with the strongest desire to avoid pregnancy are the most consistent contraceptive users [8], while those who express ambivalence about pregnancy are more likely to report inconsistent method use and discontinuation [6,9-11]. Other studies suggest few differences in contraceptive method preference between those who profess happiness about a pregnancy they would classify as unintended, and those who profess unhappiness about such a pregnancy [12]. We know much less, however, about whether emotions towards a hypothetical pregnancy, pregnancy intentions, and the importance of pregnancy prevention, are independently associated with actual contraceptive method selection and uptake. As such, this study represents a rigorous empirical test of the utility of the PATH questions.

The goal of client-centered contraceptive counseling is a shared decision-making process. The process is designed to identify contraceptive methods that are in line with patient preferences, goals, and values as well as meeting emotional and physical health needs and addressing financial limitations [13]. Clinicians provide information regarding method efficacy, side effects, duration of typical use, etc. While this information can a inform a 
client's decisionto initiate a specific method, that decision is entirely theirs to make [14]. Rooted in client-centered counseling, the HER Salt Lake Contraceptive Initiative did not specifically seek to increase use of a particular type of contraceptive method, but did find a shift toward more effective methods after the removal of costs [15]. In this analysis, we explore women's responses to the survey adapted PATH questions about attitudes towards a hypothetical pregnancy, pregnancy timing, and importance of pregnancy prevention and test associations with contraceptive method selection.

\section{Materials and Methods:}

\subsection{Enrollment}

The HER Salt Lake Contraceptive Initiative (HER Salt Lake), a prospective cohort study of individuals presenting to participating family planning clinics in Salt Lake County, Utah, provided data for this study. Throughout the initiative, trained clinic staff engaged in a contraceptive counseling conversation with each client based on ten evidence-based best practices [16]. Patients' values, life experiences, and culture were central to the shareddecision making model $[5,16]$ which was initiated as the counseling method of choice across all clinics before the start of our study.

Details on the initiative, including clinic services and data collection protocols, have been previously reported [15]. Over the 18-month enrollment period (6-month control period and 12-month intervention), a total of 11,509 individuals presented to receive new contraceptive services at HER-participating health centers and 38\% enrolled in the survey-arm ( $n=4,425$ for all enrollment periods combined, $n=3,704$ for the no-cost intervention periods). We collected surveys at baseline and eight additional time points over 36 months following receipt of initial contraceptive services.

To qualify for inclusion, women presented to one of the participating clinics for new contraceptive services, which included existing patients seeking a new contraceptive method and new patients seeking contraception at the clinic for the first time. Additional inclusion criteria for the survey-arm included being aged 18 to 45, fluent in English or Spanish, desiring to prevent pregnancy for at least one year, and possession of a functioning mobile phone at the time of the qualifying visit. The current study relied on the baseline survey that participants completed at enrollment. This analysis is restricted to individuals who enrolled during the 12-month intervention period when eligible patients received no-cost contraception.

\subsection{Sample}

For this analysis, we included eligible women who completed the enrollment survey during the intervention periods $(n=3,704)$. We excluded those with missing data for any of the PATH questions including pregnancy intentions, prevention, and happiness questions. We also excluded individuals with missing data on contraceptive method selected at baseline and those who reported they wanted to become pregnant in the next year. Of the 3,704 women who enrolled during the intervention periods, we excluded 32 because they intended to become pregnant in the next year, 42 who did not provide complete information on 
pregnancy intentions (which includes 16 with missing information and 26 who respond "other"), 158 due to missing information on the question about the importance of preventing a pregnancy, and 115 due to missing information about emotional orientation towards a hypothetical pregnancy. We excluded three participants because of missing data on the outcome measure of contraceptive method choice. Due to missing information on the demographic and socioeconomic covariates included in the final models, we excluded another 224 respondents. These 224 participants do not differ in pregnancy intentions, but place less importance on preventing a pregnancy, and would be happier about a hypothetical pregnancy. The youngest respondents, those with less education, and those who were parttime students were more likely to have missing data, especially for household income. The analytical sample consisted of 3,130 participants. Sample characteristics for demographic and socioeconomic covariates are presented in Table 1. Our sample was predominantly white, young, and low-income. The majority of participants had at least some college education, were either married or living with a partner, and most had never been pregnant.

\subsection{Key Measures}

Our outcome of interest was contraceptive method choice. We aggregated methods into two categories: 1) long-acting reversible contraception (LARC), which was comprised of the contraceptive implant, non-hormonal IUD, and hormonal IUD; and 2) other methods, which included all other non-LARC methods (i.e., oral contraceptive pills, vaginal ring, patch, contraceptive injection, and condoms). Our covariates draw on measures from the PATH (Pregnancy Attitudes, Timing, How important is prevention) approach [5]. We adjusted survey wording for use in a self-administered survey rather than an interactive clinical setting and with input from the tool and its developer, Patty Cason. The PATH questions (https://www.envisionsrh.com/path-questions-examples) are designed for a clinician to better understand their patient's needs and reproductive goals and guide a patient-centered contraceptive counseling discussion, as appropriate. The PATH Questions are intended to facilitate a conversation between patient and provider, and are framed to assist patients in clarifying their attitudes towards reproduction and to discuss their goals in the timeframe most relevant to them. The set of items below represent the survey-adapted PATH questions.

Pregnancy attitudes are represented by a question about happiness towards a hypothetical pregnancy, which we assessed with a question used in a previous study [17]. "How would you feel about getting pregnant in the next month, with response options ranging from 0 with the anchor of "worst feeling you can imagine" to 100 with the anchor of "happiest you could possibly feel." We measured pregnancy timing by the question, "What are your future pregnancy plans?" Response options included: "I would like to get pregnant in the next 2-5 years, but not in the next year"; I would like to get pregnant in the next 5-10 years, but not before then"; "I am uncertain if or when I would like to become pregnant"; "I do not plan on getting pregnant at any time in the future." We assessed importance of pregnancy prevention using the question, "How important is it to you to not get pregnant until you are ready?" (for those who intend to become pregnant at some point in the future) and "How important is it to you to not get pregnant now or in the future?" (for individuals who do not plan on getting pregnant in the future). The electronically-adapted visual analog scale (VAS) ranged from 0 with the anchor of "not at all important" to 100 with the anchor of "the most important." 


\subsection{Analytical approach}

We described contraceptive uptake patterns and pregnancy intentions in our sample. We further summarized reported pregnancy attitudes, timing and happiness toward a hypothetical pregnancy and explored the bivariate association between the PATH questions and contraceptive method uptake. We used multivariable Poisson regression analyses with robust standard errors to examine the relationship between contraceptive uptake and pregnancy intentions (Model 1), which most closely represents the One Key Question ${ }^{\mathrm{TM}}$ approach. In Models $2-4$, we included the individual PATH questions separately and jointly. In step-wise models we explore the inclusion of age, race, education, parity, insurance status, sexual identity, relationship status, enrollment period, and clinic location, based on their possible association with method choice. We excluded age and relationship status from our models, as they were significantly associated with method choice at the imposed minimum significance threshold of $\mathrm{p}<.10$. We reported prevalence ratios (PR) and calculated predicted probabilities of choosing LARC based on Models 2 and 4 to illustrate substantive associations between PATH and contraceptive choice. We used Stata v15.0 or higher for all analyses.

\section{Results}

A majority of clients (62\%) selected either an IUD or implant at the time of enrollment. Overall, $24 \%$ of participants intended to get pregnant in 2-5 years, and 30\% in 5-10 years. About $20 \%$ of participants were uncertain about when they intended to get pregnant and $26 \%$ of participants intended to never get pregnant.

We found lower importance of pregnancy prevention for those with short-term (2-5 years) pregnancy timing goals compared to those with longer-term or uncertain pregnancy timing (see Table 2). We also found that importance of pregnancy prevention was lowest among those who never intended to get pregnant in the future. Table 2 also shows that those who intended to get pregnant within five years reported higher happiness scores towards a pregnancy in the next month compared to all other respondents. The correlation between importance of pregnancy prevention and emotions towards a pregnancy in the next month was small to moderate at $\mathrm{r}=-.19$ (95\% CI based on bootstrap estimation with 100 repetitions -0.22 to -0.15$)$.

In adjusted models (Table 3), we found that the PR of choosing IUDs or implants were significantly higher for individuals with all other pregnancy intentions compared to those who intend pregnancy in the next 2-5 years (Model 1; aPRnever=1.16 95\% CI 1. 07-1.26; aPR5-10 years $=1.14$ 95\% CI 1.05-1.24; aPRuncertain $=1.1995 \%$ CI 1.09-1.30). Next, we explored the extent to which pregnancy attitudes and attitudes towards prevention are associated with LARC selection. Happiness towards a hypothetical pregnancy was significantly associated (Model 2, aPR=0.97 95\% CI 0.95-0.99). Reported importance of pregnancy prevention was not associated with contraceptive choices (Model 3). In Model 4 we estimated an adjusted PATH model that includes pregnancy attitudes and timing. Effect sizes remained similar when we considered both measures in the same model. In the adjusted PATH model (model 4), similar to models 1, shorter-term pregnancy intentions were associated with lower uptake of LARC methods compared to all other intentions 
$\left(\mathrm{aPR}_{\text {never }}=1.1395 \% \mathrm{CI} 1.10-1.23 ; \mathrm{aPR}_{5-10}\right.$ years $=1.1295 \%$ CI $1.03-1.22 ; \mathrm{aPR}_{\text {uncertain }}=1.16$ 95\% CI 1.06-1.27). Even when we included intended pregnancy timing in the model, attitudes towards pregnancy were significantly associated with LARC uptake. A 10-point increase on a 100 VAS for attitudes towards a hypothetical pregnancy was associated with a decrease in the rate of LARC selection by 0.98 (PR 95\% CI 0.96-0.999).

To better assess the clinical relevance of the small effect sizes, we calculated predicted probabilities of choosing LARC based on Model 4. Figure 1 illustrates the size of this effect. A hypothetical patient who wants to become pregnant within 5 years, but has the most negative feelings about a pregnancy right now, has a higher probability of choosing a LARC method compared to a person who never wants to be pregnant but has the most positive feelings towards an unintended pregnancy.

\section{Discussion}

We found that the chances of choosing LARC were reduced for individuals who would like to get pregnant in the next 2-5 years compared to those who were either not desiring a future pregnancy, who were uncertain, or who intended becoming pregnant in 5-10 years. We did not find an independent effect of the importance of pregnancy prevention on method choice. However, we found a small independent effect of reported happiness about a hypothetical pregnancy on contraceptive method choice. While effects were small, this finding supports previous published research and clinical anecdotes suggesting that a client's emotions towards a potential pregnancy matter. In research and client-centered contraceptive care the more nuanced pregnancy preferences should be acknowledged, not merely the cognitive plans for pregnancy timing. While streamlined approaches to integrate questions surrounding pregnancy risk into primary care and medical subspecialties is critical, reliance on single-dimension measure like One Key Question [4] might not be sufficient in understanding a patient's emotional orientation, nuanced pregnancy intentions, and contraceptive needs.

Our study population is limited to patients of participating family planning health centers in Salt Lake County, and findings may not be generalizable to other health care settings. Because of our study design, which sampled individuals seeking contraceptive care, we cannot make conclusive statements about the extent to which attitudes about importance of pregnancy prevention influence contraceptive choices in non-contraceptive visits. Because a non-trivial number of participants had missing data on key covariates, especially household income, our sample might overrepresent slightly older individuals with more stable lives. Additional research is needed to better understand contraceptive choices among those who intend to "never" become pregnant. Moreover, the wording of questions about pregnancy attitudes and importance of pregnancy prevention differ in the referenced time frame: the former refers to attitudes about a hypothetical pregnancy in the next month, the latter queries the importance of a preventing a pregnancy until a respondent is ready (or "ever" for those who never indent to become pregnant). However, our study has several strengths. Unlike most research seeking to identify predictors of contraceptive method choice, our study is prospective and tests the independent effect of the different PATH dimensions. Our research design further eliminated any financial constraints from contraceptive choices. 
While providers have long considered pregnancy intentions central to understanding contraceptive choices, these data highlight the complexity of individual's PATH orientations toward pregnancy preferences. For individuals who would like to become pregnant in the next $2-5$ years, patient options could be expanded with the discussion about the possibility of shorter-term use of LARC, in addition to a detailed discussion of all short-acting methods and return to fertility. Individuals who indicate they would be very happy about becoming pregnant in the short term may benefit from information on preconception care, in addition to contraceptive counseling.

When addressing the needs of individuals who report they do not want to get pregnant in the next year, a clinical encounter can still be used for preconception education. Patients may be interested in learning more about return to fertility for both long-acting methods and shortacting methods [18]. Future research on PATH questions should explore their association with a variety of contraceptive choices and methods mix patterns. Additionally, future research should explore how pregnancy attitudes and timing might interact with the importance of pregnancy preventions, as well as emotions about a hypothetical pregnancy as they shape contraceptive behaviors jointly and separately. Expanding research questions and contraceptive counseling to consider the multiple domains of PATH questions has the potential to enhance our understanding of clients' personal pregnancy preferences.

\section{Funding:}

This project is funded by the Society of Family Planning Research Fund, the William and Flora Hewlett Foundation, and an anonymous foundation. The following companies contributed contraceptive products for the project: Bayer Women's Healthcare, Merck \& Co. Inc., and Teva Pharmaceuticals.

Use of REDCap provided by Eunice Kennedy Shriver National Institute of Child Health and Development grant (8UL1TR000105 (formerly UL1RR025764) NCATS/NIH).

Team members receive support from the Eunice Kennedy Shriver National Institute of Child Health \& Human Development and the Office of Research on Women's Health of the National Institute of Health, JNS via Award Number K12HD085852 and DKT via K24HD087436.

The content is solely the responsibility of the authors and does not necessarily represent the official view of any of the funding agencies or participating institutions, including the National Institutes of Health, the University of Utah or Planned Parenthood Federation of America, Inc.

\section{References}

[1]. Bachrach CA, Newcomer S. Intended pregnancies and unintended pregnancies: Distinct categories or opposite ends of a continuum? Fam Plann Perspect. 1999;31:251-2. [PubMed: 10723654]

[2]. Jones RK. Change and consistency in US women's pregnancy attitudes and associations with contraceptive use. Contraception. 2017;95:485-90. [PubMed: 28137557]

[3]. Aiken AR, Borrero S, Callegari LS, Dehlendorf C. Rethinking the pregnancy planning paradigm: unintended conceptions or unrepresentative concepts? Perspect Sex Reprod Health. 2016;48:147. [PubMed: 27513444]

[4]. Bellanca HK, Hunter MS. ONE KEY QUESTION : Preventive reproductive health is part of high quality primary care. Contraception. 2013;88:3-6. [PubMed: 23773527]

[5]. Callegari LS, Aiken AR, Dehlendorf C, Cason P, Borrero S. Addressing potential pitfalls of reproductive life planning with patient-centered counseling. Am J Obstet Gynecol. 2017;216:129-34. [PubMed: 27776920]

[6]. Higgins JA, Popkin RA, Santelli JS. Pregnancy ambivalence and contraceptive use among young adults in the United States. Perspect Sex Reprod Health. 2012;44:236-43. [PubMed: 23231331] 
[7]. Schwarz EB, Lohr PA, Gold MA, Gerbert B. Prevalence and correlates of ambivalence towards pregnancy among nonpregnant women. Contraception. 2007;75:305-10. [PubMed: 17362711]

[8]. Jones RK, Tapales A, Lindberg LD, Frost J. Using longitudinal data to understand changes in consistent contraceptive use. Perspect Sex Reprod Health. 2015;47:131-9. [PubMed: 26287965]

[9]. Higgins JA, Hirsch JS, Trussell J. Pleasure, prophylaxis and procreation: a qualitative analysis of intermittent contraceptive use and unintended pregnancy. Perspect Sex Reprod Health. 2008;40:130-7. [PubMed: 18803794]

[10]. Zabin LS. Ambivalent feelings about parenthood may lead to inconsistent contraceptive use--and pregnancy. Perspect Sex Reprod Health. 1999;31:250.

[11]. Frost JJ, Singh S, Finer LB. Factors associated with contraceptive use and nonuse, United States, 2004. Perspect Sex Reprod Health. 2007;39:90-9. [PubMed: 17565622]

[12]. Aiken AR, Dillaway C, Mevs-Korff N. A blessing I can't afford: factors underlying the paradox of happiness about unintended pregnancy. Social Science \& Medicine. 2015;132:149-55. [PubMed: 25813729]

[13]. Secura GM, Allsworth JE, Madden T, Mullersman JL, Peipert JF. The Contraceptive CHOICE Project: reducing barriers to long-acting reversible contraception. Am J Obstet Gynecol. 2010;203:115 e1-. e7. [PubMed: 20541171]

[14]. Dehlendorf C, Grumbach K, Schmittdiel JA, Steinauer J. Shared decision making in contraceptive counseling. Contraception. 2017;95:452-5. [PubMed: 28069491]

[15]. Sanders JN, Myers K, Gawron LM, Simmons RG, Turok DK. Contraceptive method use during the community-wide HER salt lake contraceptive initiative. Am J Public Health. 2018;108:5506. [PubMed: 29470119]

[16]. Dehlendorf C, Krajewski C, Borrero S. Contraceptive counseling: best practices to ensure quality communication and enable effective contraceptive use. Clinical obstetrics and gynecology. 2014;57:659. [PubMed: 25264697]

[17]. Aiken AR, Potter JE. Are Latina women ambivalent about pregnancies they are trying to prevent? Evidence from the Border Contraceptive Access Study. Perspect Sex Reprod Health. 2013;45:196-203. [PubMed: 24192284]

[18]. Higgins JA. Pregnancy Ambivalence and Long-Acting Reversible Contraceptive (LARC) Use Among Young Adult Women: A Qualitative Study. Perspect Sex Reprod Health. 2017. 


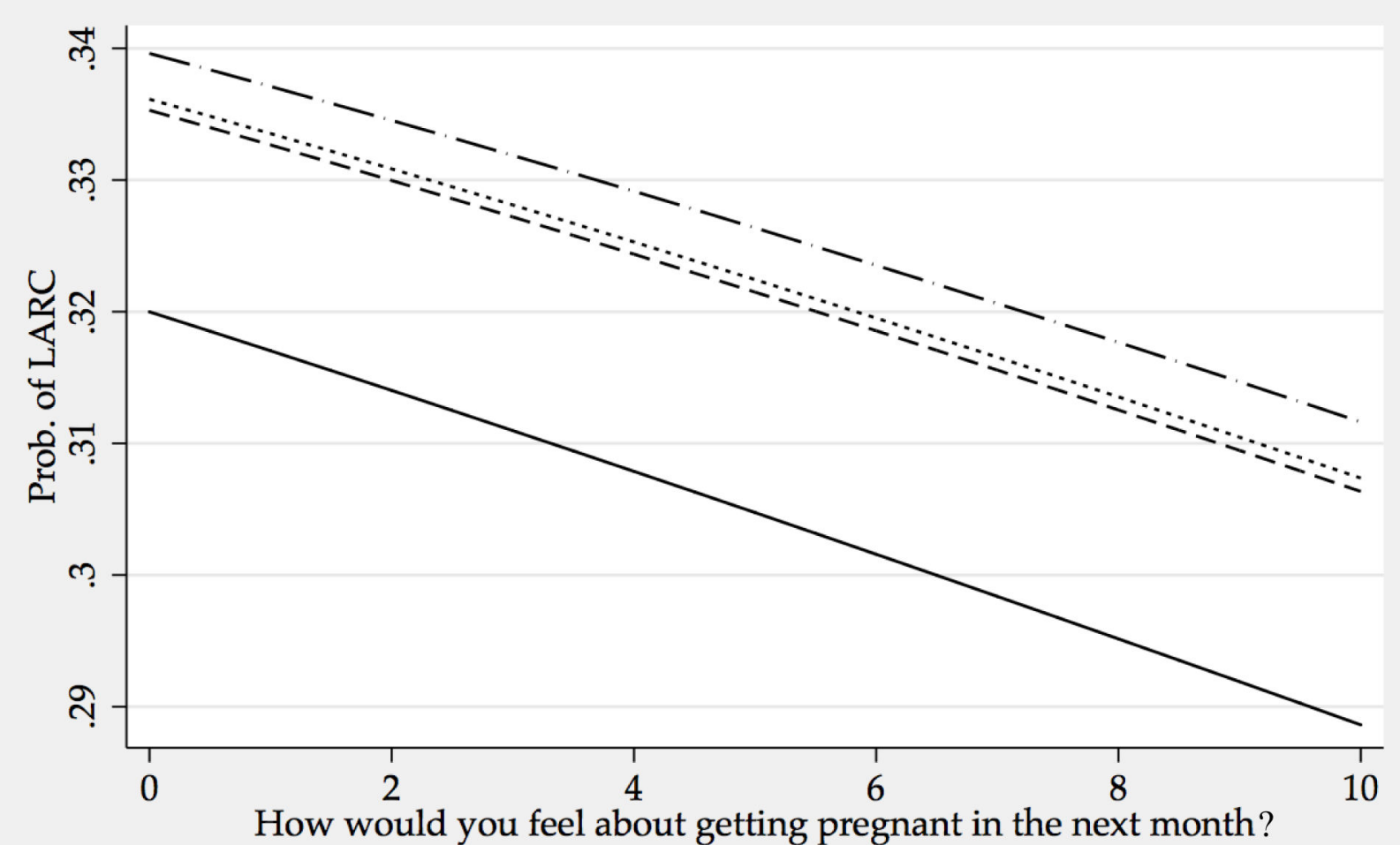

Worst

feeling ever

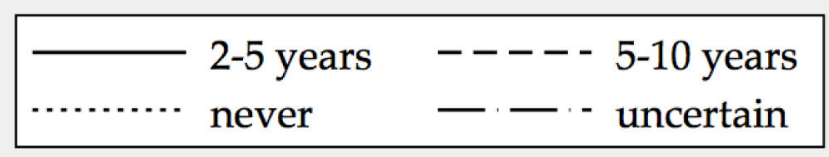

Best feeling

ever

\section{Figure 1.}

: Predicted probability of LARC choice by pregnancy intention and emotions towards pregnancy based on Poisson Regression Models (Table 3) for participants who enrolled in the survey arm of the HER Salt Lake Contraceptive Initiative and responded to key attitude, sociodemographic, and behavior measures.

Note: N=3130. Based on results from multivariable Poisson regressions that adjusted for race, education, parity, insurance status, sexual identity, enrollment period, and clinic location. All controls held at sample means 
Table 1:

Sample Characteristics of participants who enrolled in the survey arm of the HER Salt Lake Contraceptive Initiative and responded to key attitude, sociodemographic, and behavior measures $(\mathrm{N}=3,130)$.

\begin{tabular}{|c|c|c|c|c|}
\hline & Percent/Mean (n) & Std. Dev. & Min & $\operatorname{Max}$ \\
\hline \multicolumn{5}{|l|}{ Race/Ethnicity } \\
\hline White (non-Hispanic) & $65 \%(2,155)$ & & 0 & 1 \\
\hline Hispanic (any Race) & $23 \%(765)$ & & 0 & 1 \\
\hline Non-White (non-Hispanic) & $12 \%(414)$ & & 0 & 1 \\
\hline Income as percent of federal poverty line & $159(3,130)$ & 284 & 0 & 12626 \\
\hline \multicolumn{5}{|l|}{ Study Period } \\
\hline Intervention 1 & $49 \%(1,640)$ & & 0 & 1 \\
\hline Intervention 2 & $51 \%(1,714)$ & & 0 & 1 \\
\hline \multicolumn{5}{|l|}{ Currently has Health Insurance } \\
\hline No & $53 \%(1,773)$ & & 0 & 1 \\
\hline Yes & $46 \%(1,581)$ & & 0 & 1 \\
\hline \multicolumn{5}{|l|}{ Level of Education } \\
\hline High School or Less & $43 \%(1,422)$ & & 0 & 1 \\
\hline Some College or More & $57 \%(1,908)$ & & 0 & 1 \\
\hline \multicolumn{5}{|l|}{ Current School Enrollment } \\
\hline Not in school & $66 \%(2,185)$ & & 0 & 1 \\
\hline Enrolled Part-time & $12 \%(386)$ & & 0 & 1 \\
\hline Enrolled Full-time & $23 \%(752)$ & & 0 & 1 \\
\hline \multicolumn{5}{|l|}{ Sexual Identity } \\
\hline Exclusively Heterosexual & $71 \%(2,355)$ & & 0 & 1 \\
\hline Mostly Heterosexual & $16 \%(511)$ & & 0 & 1 \\
\hline Bisexual & $12 \%(386)$ & & 0 & 1 \\
\hline Mostly or Exclusively Gay/Lesbian & $1 \%(20)$ & & 0 & 1 \\
\hline Other & $1 \%(25)$ & & 0 & 1 \\
\hline \multicolumn{5}{|l|}{ Number of Previous Pregnancies } \\
\hline 0 & $63 \%(2,079)$ & & 0 & 1 \\
\hline 1 & $16 \%(513)$ & & 0 & 1 \\
\hline 2 & $9 \%(313)$ & & 0 & 1 \\
\hline 3 or more & $12 \%(402)$ & & 0 & 1 \\
\hline \multicolumn{5}{|l|}{ Enrollment Location } \\
\hline Clinic A (Abortion clinic) & $15 \%(499)$ & & 0 & 1 \\
\hline Clinic B (Title X clinic) & $33 \%(1,113)$ & & 0 & 1 \\
\hline Clinic C (Title X clinic) & $31 \%(1,055)$ & & 0 & 1 \\
\hline Clinic D (Title X clinic) & $20 \%(687)$ & & 0 & 1 \\
\hline
\end{tabular}


Table 2:

Descriptive Statistics for participants who enrolled in the survey arm of the HER Salt Lake Contraceptive Initiative and responded to key attitude, sociodemographic, and behavior measures.

\begin{tabular}{|c|c|c|c|c|c|c|}
\hline & \multicolumn{2}{|c|}{$\begin{array}{l}\text { Attitudes towards importance of pregnancy } \\
\text { prevention } 1\end{array}$} & & \multicolumn{3}{|c|}{ Emotions about a hypothetical pregnancy 1} \\
\hline & Mean & $95 \% \mathrm{CI}$ & & Mean & $95 \%$ & \\
\hline $\begin{array}{l}\text { Full Sample By Pregnancy } \\
\text { ntentions (\% of sample) }\end{array}$ & $86(\mathrm{SD}=21)^{2}$ & 85 & 87 & $15(\mathrm{SD}=17)^{2}$ & 14 & 16 \\
\hline $2-5$ years $(24 \%)$ & 84 & 83 & 86 & 25 & 23 & 26 \\
\hline $5-10$ years $(30 \%)$ & 90 & 89 & 90 & 13 & 12 & 14 \\
\hline Uncertain $(20 \%)$ & 89 & 88 & 91 & 12 & 11 & 14 \\
\hline Never (26\%) & 80 & 78 & 82 & 11 & 10 & 12 \\
\hline
\end{tabular}

$\mathrm{N}=3,130$ Based on 100-point visual analog scale (VAS). Higher scores indicate greater importance of prevention and more positive emotions towards pregnancy.

IRange from 0 to 100 .

2 Multivariate models show effects in 10-point units 
Table 3:

Prevalence Ratios of choosing LARC based on Poisson Regression Models among participants who enrolled in the survey arm of the HER Salt Lake Contraceptive Initiative and responded to key attitude, sociodemographic, and behavior measures.

\begin{tabular}{|c|c|c|c|c|}
\hline VARIABLES & $\begin{array}{l}\text { Model } 1 \text { Pregnancy } \\
\text { Intentions/Timing }\end{array}$ & $\begin{array}{c}\text { Model } 2 \\
\text { Pregnancy Attitudes }\end{array}$ & $\begin{array}{l}\text { Model } 3 \text { How } \\
\text { Important Is } \\
\text { Prevention? }\end{array}$ & $\begin{array}{c}\text { Model } 4 \text { Pregnancy Attitudes } \\
\text { + Timing }\end{array}$ \\
\hline \multicolumn{5}{|l|}{ Pregnancy Intentions } \\
\hline $2-5$ years & Ref. category & & & Ref. category \\
\hline \multirow[t]{2}{*}{ Never } & $1.16^{* * *}$ & & & $1.13^{* * *}$ \\
\hline & $(1.07-1.26)$ & & & $(1.04-1.23)$ \\
\hline \multirow[t]{2}{*}{$5-10$ years } & $1.142^{* * *}$ & & & $1.122^{* * * *}$ \\
\hline & $(1.05-1.24)$ & & & $(1.03-1.22)$ \\
\hline \multirow[t]{2}{*}{ Uncertain } & $1187^{* * * *}$ & & & $1.16^{* * * *}$ \\
\hline & $(1.09-1.30)$ & & & $(1.06-1.27)$ \\
\hline Hypothetical & & $097^{* * * *}$ & & $0.98^{* *}$ \\
\hline Pregnancy & & $(0.95-0.99)$ & & $(0.96-0.999)$ \\
\hline \multicolumn{5}{|l|}{ (10 point change on } \\
\hline \multicolumn{5}{|l|}{ VAS) } \\
\hline Importance of & & & 1.004 & \\
\hline Pregnancy Prevention & & & $(0.99-1.02)$ & \\
\hline \multicolumn{5}{|c|}{$(10 \text { point change on VAS })^{1}$} \\
\hline Observations & 3,130 & 3,130 & 3,130 & 3,130 \\
\hline
\end{tabular}

Note: $\mathrm{N}=3,130$, adjusted for race (white respondents have higher LARC uptake than nonwhite respondents, education (+, indicates positive association with LARC uptake at .05 level of higher), parity (+), insurance status (-), sexual identity, enrollment period $3(+$, vs. period 2$)$, and clinic location (negatively associated with LARC uptake for all clinic locations compared to abortion clinic); Numbers in parentheses are $95 \%$ confidence interval based on robust standard errors;

****

$\mathrm{p}<0.001$,

* $\mathrm{p}<0.01$,

p $<0.05$;

$1_{\text {see Table } 2}$ 